\begin{abstract}
A new class of model-based filters for extracting trends and cycles in economic time series is presented. These low pass and band pass filters are derived in a mutually consistent manner as the joint solution to a signal extraction problem in an unobserved components model. The resulting trends and cycles are computed in finite samples using the Kalman filter and associated smoother. The filters form a class which is a generalisation of the class of Butterworth filters, widely used in engineering. They are very flexible and have the important property of allowing relatively smooth cycles to be extracted from economic time series. Perfectly sharp, or ideal, band pass filters emerge as a special case.

Applying the method to a quarterly series on US investment shows a clearly defined cycle currently at the peak of a boom.

KEYWORDS: Band pass filter, Butterworth filter, ideal filter, Kalman filter, signal extraction, unobserved components.

JEL classification: $\mathrm{C} 15, \mathrm{C} 22$.
\end{abstract}




\title{
General model-based filters for extracting cycles and trends in economic time series
}

\author{
Andrew C. Harvey and Thomas M. Trimbur \\ Faculty of Economics and Politics, Cambridge University
}

July 9, 2001

\section{Introduction}

Separating out trends and cycles is fundamental to much of macroeconomic analysis. For example, the filter introduced by Hodrick and Prescott (1997) is widely used for this purpose. More generally trend extraction may be carried out by using a low-pass Butterworth filter. This class of filters is widely used in engineering, but, as recently pointed out by Gomez (2000), the Hodrick-Prescott (HP) filter is a special case.

Rather than defining the cycle simply as the residual part of the series after a trend has been extracted, a band pass filter may be used to remove high frequency as well as low frequency components. In a recent paper Baxter and King (1999) consider the design of band pass filters and their implementation in finite samples. Their prime concern is to approximate the 'ideal' filter (a perfectly sharp band pass filter) in the time domain using a finite moving average and to use this filter for extracting business cycles. Christiano and Fitzgerald (1999) also regard the rectangular gain function as the ultimate goal of filtering operations; they too propose a finite sample approximation, demonstrating its properties when applied to US macroeconomic data. The ideal filter can be obtained as the limiting case of a Butterworth band pass filter.

An alternative approach is to extract trends and cycles from unobserved components, or structural, time series models. The parameters of such models are typically estimated by maximum likelihood and, once this has been done, optimal estimates of the components are obtained by smoothing algorithms. The calculations are most easily performed by putting the model in state space form; see Harvey (1989). The principal aim of this article is to extend the class of structural time series models so that they are able to extract smoother cycles. In doing so, it is shown that the implied filters are closely linked to Butterworth filters and that a model yielding the equivalent of an ideal band pass filter can be obtained as a special case.

The attraction of the model-based approach is that the filters implicitly defined by the model are consistent with each other and with the data. Furthermore they automatically adapt to the ends of the sample and, if desired, 
root mean square errors can be calculated. The models can also be used to gain insight into the more $a d$ hoc filters used in business cycle analysis, indicating when it might be appropriate to use them and when they can lead to serious distortions of the kind documented by Cogley and Nason (1995) and Harvey and Jaeger (1993) for the HP filter and by Murray (2000) and Benati (2000) for ideal band pass filters.

The plan of the article is as follows. Section 2 begins by discussing the frequency domain properties of the Butterworth class of low pass and band pass filters. Section 3 then sets out classes of unobserved components models for which the Butterworth filters are optimal, at least for estimating components near the middle of a long sample. The class of band pass Butterworth filters is extended by basing them on models containing stationary cycles. This is crucial for economic applications. A general class of unobserved components models, containing both trends and cycles, is defined in section 4 . The implied filters for extracting trends and cycles from such models are mutually consistent and the way in which they interact is studied in the frequency domain. Section 5 applies the new models to real macroeconomic time series, with particular emphasis on the extraction of relatively smooth cycles. Section 6 concludes.

\section{Low pass and high pass filters}

Low pass filters are used to isolate the trend in a series. Viewed in the frequency domain, the effect of such filters is to pick out the low frequency movements. In a similar way, band pass filters are designed to focus on mid-range frequencies, associated with movements such as business cycle fluctuations.

The low pass Butterworth filter depends on a positive parameter, $q$, and a positive integer index $m$. It can be expressed in terms of the lag operator, $L$, as follows:

$$
B_{m}^{l p}(L)=\frac{1}{1+q^{-1}|1-L|^{2 m}}, \quad m=1,2,3, . .
$$

Expanding this expression in powers of $L$ gives the coefficients, $w_{j}$, attached to the adjacent observations when the trend is estimated in the middle of a long sample, that is

$$
B_{m}^{l p}(L) y_{t}=\sum_{j} w_{j} y_{t+j}
$$

For example if $m=1$, it can be shown that the weights die away exponentially on either side, that is

$$
w_{j}=\{(1+\theta) /(1-\theta)\}(-\theta)^{|j|}, \quad j=0,1,2, \ldots .
$$

where $\theta=\left\{-q-2+\sqrt{q^{2}+4 q}\right\} / 2$. The weights in the filter sum to one, as can be seen by setting $L=1$ in (1), while the corresponding detrending filter is given by $1-B_{m}^{l p}(L)$. 


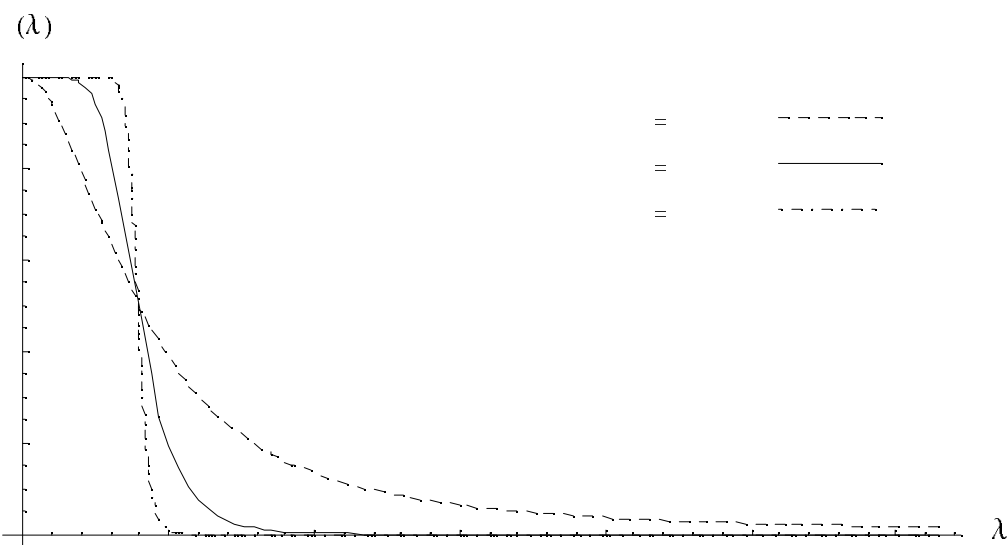

Figure 1: Butterworth low pass filter, $B_{m}^{l p}(\lambda)$, for $\lambda_{l p}=\pi / 8$ and $m=1,3$ and 10.

The form of the Butterworth filter is motivated by its properties in the frequency domain. The effect of the filter can be obtained from the frequency response function, which is found by replacing $L$ by $\exp (-i \lambda)$ in (1). The gain is the modulus of the frequency response function. Assuming the original series to be stationary, the gain shows how the amplitude of components at each frequency are affected; see, for example, Harvey (1993, p 190). Squaring the gain gives the factor by which the spectrum of the original series must be multiplied to give the spectrum of the filtered series. Since the filter in (1) is symmetric and the frequency response function is nowhere negative, the gain is equal to the frequency response function. Writing $B_{m}^{l p}\left(e^{-i \lambda}\right)$ somewhat more compactly as $B_{m}^{l p}(\lambda)$, the gain can be expressed as

$$
B_{m}^{l p}(\lambda)=\frac{1}{1+q^{-1}(2-2 \cos \lambda)^{m}}
$$

Using standard trigonometric identities, it can be shown that

$$
B_{m}^{l p}(\lambda)=\frac{1}{1+q^{-1} 2^{2 m} \sin ^{2 m}(\lambda / 2)}=\left[1+\left(\frac{\sin (\lambda / 2)}{\sin \left(\lambda_{l p} / 2\right)}\right)^{2 m}\right]^{-1}
$$

where

$$
q=\left[2 \sin \left(\lambda_{l p} / 2\right)\right]^{2 m}
$$


In the second equation in (5), $\lambda_{l p}$ is the frequency at which the gain equals one-half. Without loss of generality in the gain shape, we can restrict attention to the case $0<\lambda_{l p} \leq \pi$ since the function $\sin ^{2}(x / 2)$ has a period of $2 \pi$ and is symmetric. The low-pass filter focuses on fluctuations with frequency below $\lambda_{l p}$. As can be seen in figure 1 , where $B_{m}^{l_{p}}(\lambda)$ is displayed for $m$ equal to 1,3 , and 10 with $\lambda_{l p}$ fixed at $\pi / 8$, the higher frequencies are cut off more sharply as $m$ increases. Indeed it can be seen from (5) that the filter becomes more rectangular as $m \rightarrow \infty$. The parameter $q$ also influences the sharpness and location; consistent with the relationship in (6), according to which higher values of $q$ coincide with a larger index $m$, the Butterworth low pass filter becomes sharper as $q$ increases while $\lambda_{l p}$ remains fixed. On the other hand, if the order $m$ is fixed, then higher values of $q$ are associated with increases in the cutoff frequency $\lambda_{l p}$. Overall the class of Butterworth low pass filters ${ }^{1}$ offers a high degree of flexibility with respect to the location of the cutoff frequency and the sharpness of the filter.

Cyclical components can be extracted from an observed series using bandpass filters based on the Butterworth low-pass filters. This may be done by subtracting the weights for a Butterworth low pass filter for $\lambda_{l p}=\lambda_{1}$ from those for a low-pass filter for $\lambda_{2}$ with $\lambda_{2}>\lambda_{1}$. Setting the low pass index, $m$, to $n$ yields a gain function given by

$$
B_{n}^{b p}\left(\lambda ; \lambda_{1}, \lambda_{2}\right)=\left[1+\left(\frac{\sin (\lambda / 2)}{\sin \left(\lambda_{2} / 2\right)}\right)^{2 n}\right]^{-1}-\left[1+\left(\frac{\sin (\lambda / 2)}{\sin \left(\lambda_{1} / 2\right)}\right)^{2 n}\right]^{-1}, \quad \lambda_{2}>\lambda_{1}
$$

Since the weights in the low pass filters sum to unity, the weights in the band pass filter sum to zero. As $n \rightarrow \infty$ the gain becomes rectangular, cutting out all frequencies outside the range between $\lambda_{2}$ and $\lambda_{1}$. Some business cycle researchers have argued for the desirability of such sharp filters, referring to them as ideal. The use of a sharp band pass filter involves the notion that the underlying cyclical component itself is in fact defined by a range of frequencies. Thus, in Baxter and King (1999), the business cycle component of a macroeconomic time series is equated to those components with periodicity between 6 and 32 quarters, following Burns and Mitchell (1946).

A slightly different class of band pass filters is obtained from low-pass filters by a transformation as in Oppenheim and Schaffer (1989); see also Gomez (2000). Given constants $q$ and $\lambda_{c}$, which satisfy $q>0$ and $0<\lambda_{c}<\pi$, these filters have gain functions:

$$
\begin{aligned}
B_{n}^{b p}(\lambda) & =\left[1+\frac{1}{q}\left[\frac{2\left(1+\cos 2 \lambda+2 \cos ^{2} \lambda_{c}-4 \cos \lambda_{c} \cos \lambda\right)}{\left(1+\cos ^{2} \lambda_{c}-2 \cos \lambda_{c} \cos \lambda\right)}\right]^{n}\right]^{-1} \\
& =\left[1+\frac{1}{q}\left[\frac{4\left(\cos \lambda-\cos \lambda_{c}\right)^{2}}{\left(1+\cos ^{2} \lambda_{c}-2 \cos \lambda_{c} \cos \lambda\right)}\right]^{n}\right]^{-1}
\end{aligned}
$$

\footnotetext{
${ }^{1}$ Another class of Butterworth filters is gven by replacing the sine function in equation (5) by a tangent function; see Gomez (2000). We restrict attention to sine function filters as they are most easily linked to unobserved components models.
} 
$(\lambda)$

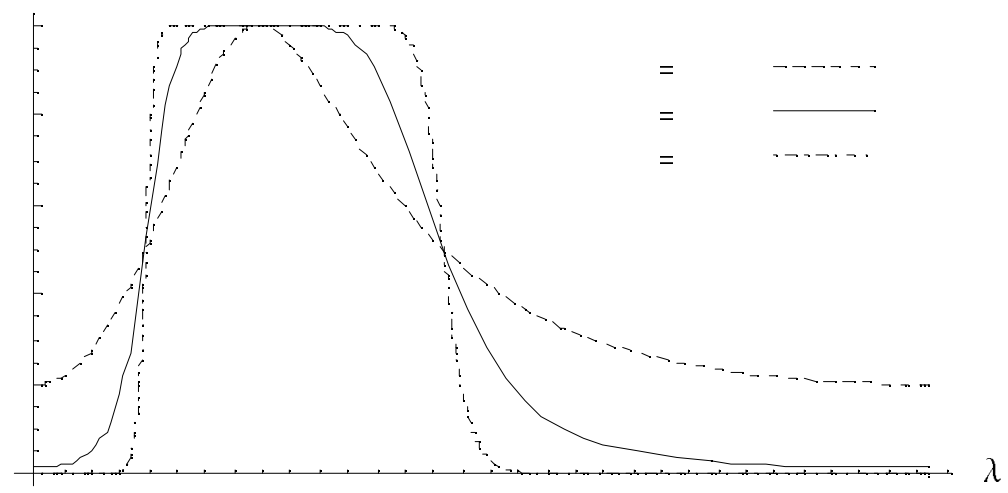

Figure 2: Butterworth band pass filter, $B_{n}^{b p}(\lambda)$, for $\lambda_{c}=\pi / 4, q=1$, and $n=1,3$ and 10 .

The filters are indexed by a positive integer $n$. We will call them band pass Butterworth filters. A plot of the gain function is shown in figure 2 for different values of $n$ with $\lambda_{c}$ set to $\pi / 4$ and $q=1$. As $n$ increases, the filter cuts out an increasingly distinct block of frequencies and so corresponds to an ideal band pass filter. Since $B_{n}^{b p}(0)=q /\left(q+4^{n}\right)$, the weights only sum to zero as $n$ goes to infinity. (Contrary to what is argued in Baxter and King (1999) in the context of an ideal filter, there is no reason why the weights for a band pass filter should sum to zero in general. However, the combined sum of the weights for extracting all the components which are not part of the trend will normally be zero.)

The parameter $\lambda_{c}$ plays the role of a central frequency as the band selected by the filter tends to concentrate in a neighborhood surrounding it. The parameter $q$ controls the spread of the gain function. Figure 3 displays the band pass Butterworth filter for different values of this parameter, with $\lambda_{c}=\pi / 4$ and $n=3$. The peak of the graph becomes perceptibly narrower as $q$ shifts from 10 to 0.1, indicating an increased focus on components with frequency near $\lambda_{c}$.

Thus, the band pass Butterworth filters possess a considerable degree of flexibility regarding those aspects of primary interest to analysts. While the general location of the peak of the gain is controlled by $\lambda_{c}$, the sharpness or rectangular resolution of the filter depends on the index $n$. The range of frequencies captured by the filter is determined by $q$. A measure of bandwidth can be found by solving for the two values of $\lambda$ at which the gain function equals $1 / 2$. These values represent the two values of $\lambda$ for which the second term in the denominator of (7) equals one. Thus, the solutions satisfy 
$(\lambda)$

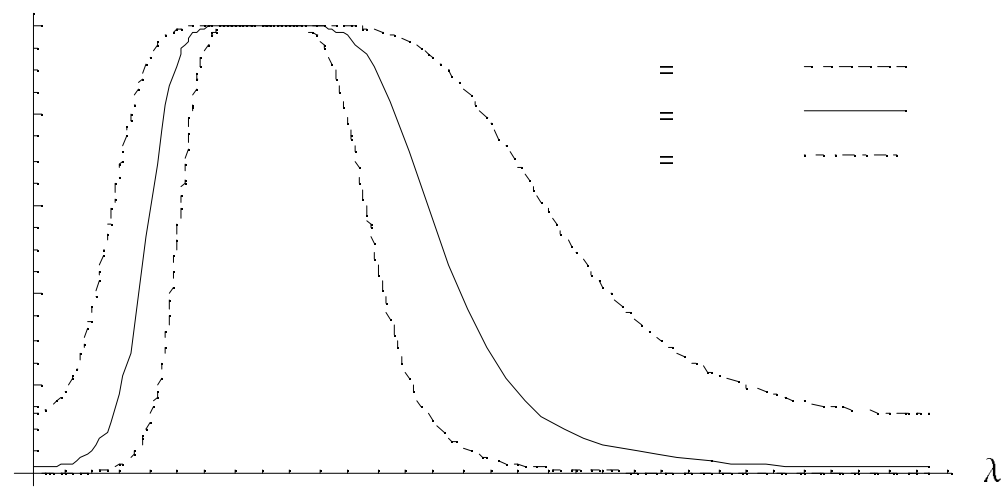

Figure 3: Butterworth band pass filter, $B_{n}^{b p}(\lambda)$, for $n=3, \lambda_{c}=\pi / 4$, and $q=0.1$, 1 and 10.

$$
\frac{\left.4 \cos ^{2} \lambda-8 \cos \lambda_{c} \cos \lambda+4 \cos ^{2} \lambda_{c}\right)}{q^{1 / n}\left(1+\cos ^{2} \lambda_{c}-2 \cos \lambda_{c} \cos \lambda\right)}=1
$$

This results in a quadratic equation in $\cos \lambda$ which when solved yields:

$$
\cos \lambda=\frac{1}{4}\left(q^{1 / n}-4\right) \cos \lambda_{c} \pm \frac{1}{4}\left(q^{1 / n}\left(q^{1 / n} \cos ^{2} \lambda_{c}-4 \sin ^{2} \lambda_{c}\right)\right)^{\frac{1}{2}}
$$

Denoting the two frequencies for which $B_{n}^{b p}(\lambda)=1 / 2$ by $\lambda_{1}$ and $\lambda_{2}$, the bandwidth is given by $\left|\lambda_{2}-\lambda_{1}\right|$. Given a desired value for the bandwidth along with the frequency of interest $\lambda_{c}$, one could solve (numerically) for $q^{1 / n}$. The index, $n$, may be chosen on the basis of the desired sharpness.

The next section shows how Butterworth filters are implicitly given by particular types of unobserved components models. This gives insight into when various filters might be appropriate. It also solves the problem of how to form weights near the beginning and end of a series and suggests how the filters may be generalised.

\section{Unobserved cyclical and trend components}

This section sets out unobserved component models in which the optimal estimates of trends and cycles are given by Butterworth filters.

Definition 1 An unobserved component $\left\{\mu_{m, t}\right\}_{t=-\infty}^{\infty}$ is an mth order stochastic trend, for positive integer $m$, if 


$$
\begin{gathered}
\mu_{\mathbf{1}, t}=\mu_{\mathbf{1 , t - 1}}+\zeta_{t} \\
\mu_{i, t}=\mu_{i, t-\mathbf{1}}+\mu_{i-\mathbf{1}, t}, \quad i=2, \ldots, m
\end{gathered}
$$

where the disturbance term $\zeta_{t}$ is serially uncorrelated with mean zero and constant variance $\sigma_{\zeta}^{2}$, denoted hereafter by writing $\zeta_{t} \sim W N\left(0, \sigma_{\zeta}^{2}\right)$.

The first order stochastic trend is a simple random walk, while for $m=2$ the trend is an integrated random walk. These specifications are frequently used in structural time series models; see Harvey (1989), Young (1984) or Kitagawa and Gersch (1996). In the integrated random walk case, $\mu_{1, t}$ is the slope.

Although these stochastic trends are nonstationary, the Wiener-Kolmogorov (WK) filter can still be used to give the optimal, or more precisely minimum mean square error linear, estimator; see Bell (1984). If the only other component is white noise, $\varepsilon_{t}$, that is

$$
y_{t}=\mu_{m, t}+\varepsilon_{t}, \quad \varepsilon_{t} \sim W N\left(0, \sigma_{\varepsilon}^{2}\right)
$$

then the WK filter for a doubly infinite series yields the low pass Butterworth filter, (1), with $q=q_{\zeta}=\sigma_{\zeta}^{2} / \sigma_{\varepsilon}^{2}$. This follows immediately on substituting repeatedly to give $\mu_{m, t}=\zeta_{t} /(1-L)^{m}$ and constructing the WK filter as the ratio of the (pseudo) autocovariance generating function (ACGF) of $\mu_{m, t}$ to that of $y_{t}$. For finite samples, the trend may be extracted by the state space smoother. Note that the estimated second-order trend is equivalent to a cubic spline while $m=3$ gives a quintic spline; see Kohn, Ansley, and Wong (1992).

The specification of stochastic cyclical components takes into account the notion that economic cycles evolve over time. This specification has been found useful in modeling cyclical behaviour in a wide variety of time series, including real GNP; see Harvey and Jaeger (1993). The definition below is a straightforward generalisation based on a recursion which generates a sequence of cycles driven by a disturbance term which impacts in such a way as to preserve continuity.

Definition 2 An unobserved component $\left\{\psi_{n, t}\right\}_{t=-\infty}^{\infty}$ is an nth order stochastic cycle, for positive integer $n$, if

$$
\begin{gathered}
{\left[\begin{array}{l}
\psi_{1, t} \\
\psi_{1, t}^{*}
\end{array}\right]=\rho\left[\begin{array}{ll}
\cos \lambda_{c} & \sin \lambda_{c} \\
-\sin \lambda_{c} & \cos \lambda_{c}
\end{array}\right]\left[\begin{array}{l}
\psi_{1, t-1} \\
\psi_{1, t-1}^{*}
\end{array}\right]+\left[\begin{array}{c}
\kappa_{t} \\
0
\end{array}\right]} \\
{\left[\begin{array}{c}
\psi_{i, t} \\
\psi_{i, t}^{*}
\end{array}\right]=\rho\left[\begin{array}{ll}
\cos \lambda_{c} & \sin \lambda_{c} \\
-\sin \lambda_{c} & \cos \lambda_{c}
\end{array}\right]\left[\begin{array}{l}
\psi_{i, t-1} \\
\psi_{i, t-1}^{*}
\end{array}\right]+\left[\begin{array}{c}
\psi_{i-1, t} \\
0
\end{array}\right], \quad i=2, \ldots, n}
\end{gathered}
$$

where $\kappa_{t} \sim W N\left(0, \sigma_{\kappa}^{2}\right)$. The parameter $\rho$ is called the damping factor and satisfies $0<\rho \leq 1$, while $0<\lambda_{c} \leq \pi$. 
The first order stochastic cycle is as described in Harvey (1989), with the variance of the disturbance term for the auxiliary process $\psi_{1, t}^{*}$ set to zero instead of $\sigma_{\kappa}^{2}$. For $n=2, \psi_{2, t}$ has a first order stochastic cycle as a driving variable so the shocks to a second order cycle are themselves periodic. The properties of the cycle are obtained by first writing

$$
\left[\begin{array}{l}
\psi_{i, t} \\
\psi_{i, t}^{*}
\end{array}\right]=\left[\begin{array}{ll}
1-\rho \cos \lambda_{c} L & -\rho \sin \lambda_{c} L \\
\rho \sin \lambda_{c} L & 1-\rho \cos \lambda_{c} L
\end{array}\right]^{-1}\left[\begin{array}{l}
\psi_{i-1, t} \\
0
\end{array}\right], \quad i=2, \ldots, n
$$

from which it follows that

$$
\psi_{i, t}=c(L) \psi_{i-\mathbf{1}, t}, \quad i=2, \ldots, n
$$

where

$$
c(L)=\frac{1-\rho \cos \lambda_{c} L}{1-2 \rho \cos \lambda_{c} L+\rho^{2} L^{2}}
$$

Since $\psi_{1, t}=c(L) \kappa_{t}$, repeated substitution yields $\psi_{n, t}=[c(L)]^{n} \kappa_{t}$ and so the autocovariance generating function (ACGF) is $\left[c(L) c\left(L^{-1)}\right]^{n} \sigma_{\kappa}^{2}\right.$. The spectral generating function is obtained by replacing $L$ by $\exp (-i \lambda)$ and so the power spectrum, for $\rho<1$, is

$$
f_{\psi}\left(\lambda ; \rho, \lambda_{c}, n\right)=\frac{\sigma_{\kappa}^{2}}{2 \pi}\left[\frac{1+\rho^{2} \cos ^{2} \lambda_{c}-2 \rho \cos \lambda_{c} \cos \lambda}{1+\rho^{4}+4 \rho^{2} \cos ^{2} \lambda_{c}-4\left(\rho+\rho^{3}\right) \cos \lambda_{c} \cos \lambda+2 \rho^{2} \cos 2 \lambda}\right]^{n}
$$

This generalises the expression for $n=1$ given in Harvey (1989, p 60) except insofar as (10) contains only one disturbance term.

If the model consists of a stochastic cycle plus white noise, that is

$$
y_{t}=\psi_{n, t}+\varepsilon_{t}, \quad \varepsilon_{t} \sim W N\left(0, \sigma_{\varepsilon}^{2}\right)
$$

then the WK filter for extracting the cycle is

$$
G B_{n}^{b p}(L)=\frac{\sigma_{\kappa}^{2} c(L)^{n} c\left(L^{-1}\right)^{n}}{\sigma_{\kappa}^{2} c(L)^{n} c\left(L^{-1}\right)^{n}+\sigma_{\varepsilon}^{2}}
$$

The frequency response function is again real and positive so the gain is

$$
G B_{n}^{b p}(\lambda ; \rho)=\frac{q_{\kappa}\left[\frac{1+p^{2} \cos ^{2} \lambda_{c}-2 p \cos \lambda_{c} \cos \lambda}{1+\rho^{4}+4 p^{2} \cos ^{2} \lambda_{c}-4\left(p+p^{3}\right) \cos \lambda_{c} \cos \lambda+2 p^{2} \cos 2 \lambda}\right]^{n}}{1+q_{\kappa}\left[\frac{1+p^{2} \cos ^{2} \lambda_{c}-2 p \cos \lambda_{c} \cos \lambda}{1+p^{4}+4 p^{2} \cos ^{2} \lambda_{c}-4\left(p+p^{3}\right) \cos \lambda_{c} \cos \lambda+2 p^{2} \cos 2 \lambda}\right]^{n}}
$$

where $q_{\kappa}=\sigma_{\kappa}^{2} / \sigma_{\varepsilon}^{2}$. The notation $G B_{n}^{b p}$ is for generalised band-pass Butterworth filter of order $n$, since when $\rho=1$, the ordinary Butterworth band pass filter, $(7)$, is obtained.

Setting $\rho$ equal to 1 in the band pass filter may be unappealing insofar as it corresponds to a nonstationary cycle. Business cycles are normally thought 
$(\lambda)$

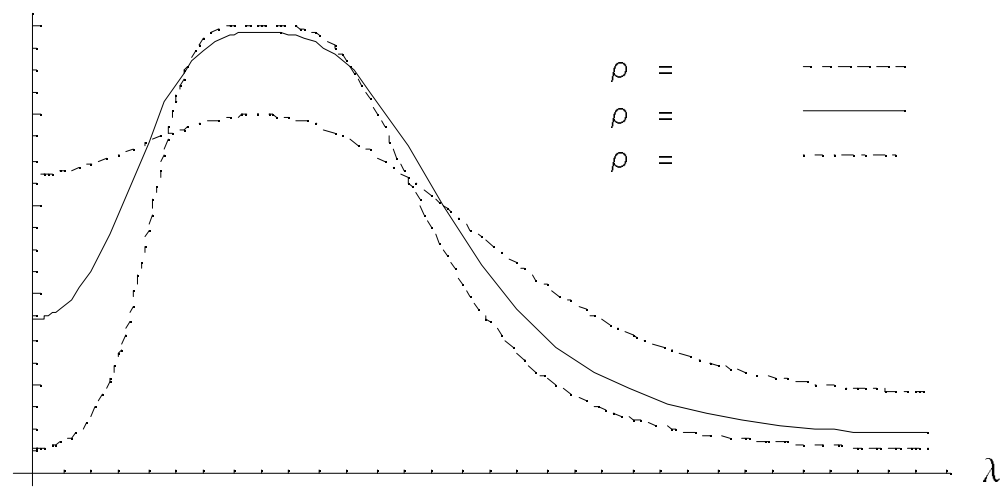

Figure 4: $G B_{n}^{b p}(\lambda ; \rho)$ for $n=2$ and $\rho=0.5,0.8$ and 1.0.

of as being stationary, so the additional flexibility resulting from the inclusion of the damping factor is an important generalisation. Figure 4 plots the gain of the band pass filter for $\rho=0.5,0.8$, and 1.0 with $n$ set to 2 . As $\rho$ becomes smaller, the gain function spreads out. Conversely, the spectrum becomes more peaked around $\lambda_{c}$ as $\rho$ increases. For $\rho=1$ the spectrum is undefined at $\lambda_{c}$, but cancellation between the numerator and denominator means that the gain is still defined.

\section{General model-based filters}

The stochastic trend and cyclical components, $\mu_{m, t}$ and $\psi_{n, t}$ defined in the last section, combine to form a class of structural time series models:

$$
y_{t}=\mu_{m, t}+\psi_{n, t}+\varepsilon_{t}, \quad t=1, \ldots T,
$$

where the irregular term $\varepsilon_{t}$ is white noise. The three components are assumed to be mutually uncorrelated; this both simplifies the statistical treatment of the model and ensures that the weighting patterns in the signal extraction filters are symmetric; see Harvey and Koopman (2000). The model can be extended so as to include a seasonal component, as in Harvey and Jaeger (1993), thereby avoiding the potentially distorting effects of seasonal adjustment procedures.

The optimal estimator of the trend (cycle) in (15) will be called the generalised low (band) pass Butterworth filter of order $(m, n)$. The low pass filter may be written

$$
G B_{m, n}^{l p}(L)=\frac{q_{\zeta} /|1-L|^{2 m}}{q_{\zeta} /|1-L|^{2 m}+q_{\kappa} /|c(L)|^{2 n}+1}
$$


$(\lambda)$

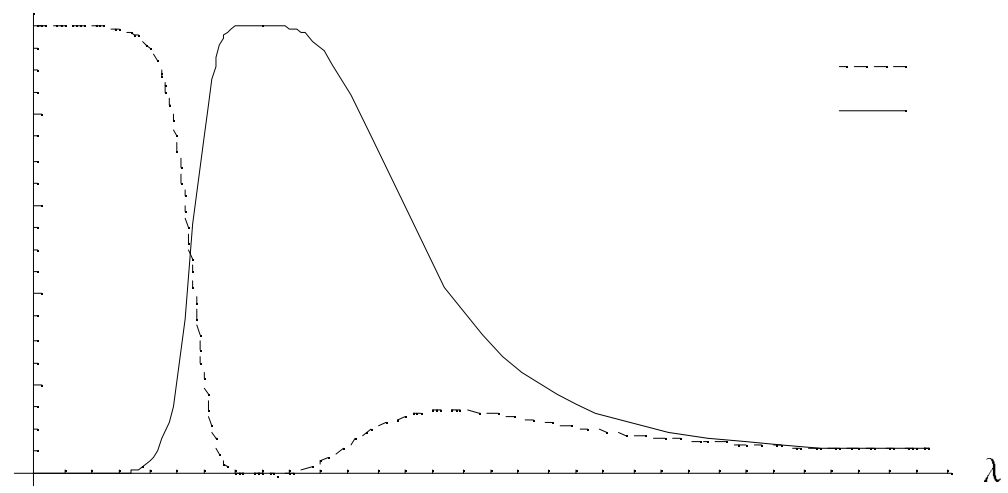

Figure 5: Generalised low pass and band pass Butterworth filters of order $(2,2)$ with $q_{\kappa}=q_{\zeta}=1$ and $\rho=1$ and $\lambda_{c}=\pi / 4$.

and its gain is

$G B_{m, n}^{l_{p}}(\lambda)=\frac{q_{\zeta}\left[\frac{1}{2-2 \cos \lambda}\right]^{m}}{q_{\zeta}\left[\frac{1}{2-2 \cos \lambda}\right]^{m}+q_{\kappa}\left[\frac{1+\rho^{2} \cos ^{2} \lambda_{c}-2 \rho \cos \lambda_{c} \cos \lambda}{1+\rho^{4}+4 \rho^{2} \cos ^{2} \lambda_{c}-4\left(\rho+\rho^{3}\right) \cos \lambda_{c} \cos \lambda+2 \rho^{2} \cos 2 \lambda}\right]^{n}+1}$

The corresponding expressions for the band pass filter, $G B_{m, n}^{b p}(L)$, are similarly obtained.

The model-based low pass and band pass filters are applied simultaneously. Applying simple low pass and band pass Butterworth filters separately will give different results. Unless the filters are 'ideal', applying individual filters consecutively will also give different results. Thus a generalised band pass Butterworth filter of order $(2,2)$ will not give the same cycle as would be obtained by applying a band pass filter of order 2 to a series which has been detrended by a low pass filter of order 2. A fully specified model enables trends and cycles to be extracted by filters which are mutually consistent. Figure 5 shows gains for the low pass and band pass Butterworth filters of order $(2,2)$, given $q_{\zeta}=q_{\kappa}=1$. There is some overlap and a comparison with figure 1 shows that the need to accommodate the band pass filter induces a dip in the low pass filter at intermediate frequencies. Figure 6 shows the same filters, but with the order increased to $(5,5)$. Both gain functions have become noticeably more block-like. Some overlap between the two still exists, but it has fallen considerably. This tendency continues as the order increases further with the gain function becoming increasingly sharp and rectangular.

The gain function shows the effect of a filter on a stationary time series. 
$(\lambda)$

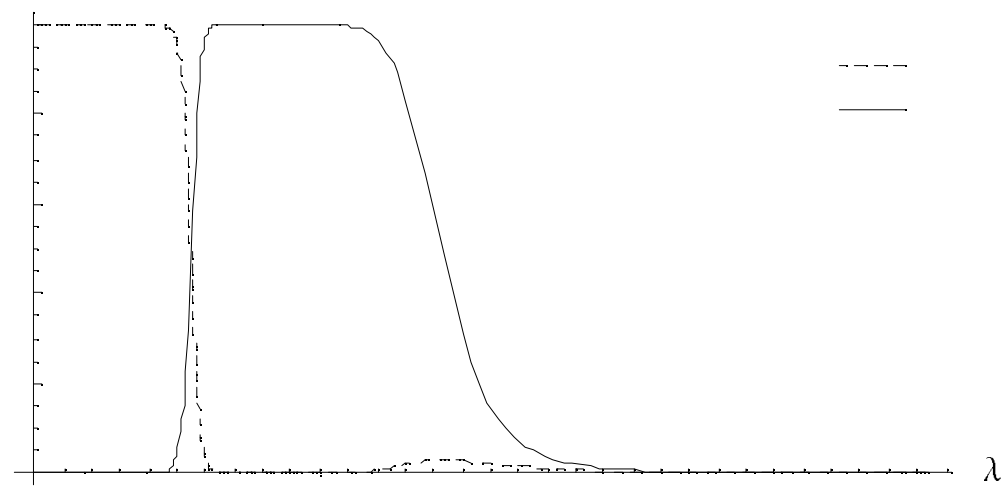

Figure 6: Generalised low pass and band pass Butterworth filters of order $(5,5)$ with $q_{\kappa}=q_{\zeta}=1$ and $\rho=1$ and $\lambda_{c}=\pi / 4$.

However, the main reason for applying filters in economics is to deal with trends, which are normally thought of as being nonstationary. The implications of a particular filter for detrending or extracting a cycle can be determined by examining the implied spectrum. For example, suppose we have an optimal filter, that is one constructed from the correct model. If the model is of the form (15), the spectrum of the extracted cycle is equal to the spectrum of the original cycle multiplied by $G B_{m, n}^{b p}(\lambda)$. Because the effect of the filter is to damp down the power of frequencies which are some way from $\lambda_{c}$, the spectrum of the extracted cycle will be more concentrated than that of the original and the series itself will be smoother.

The effect of arbitrary band pass filters can be determined by deriving the implied spectra in a similar way as was done for detrending filters in Harvey and Jaeger (1993). An important question concerns the difference between applying a simple band pass filter and a generalised band pass which takes account of the possibility of a stochastic trend. Suppose the true series is integrated of order $d$, denoted $I(d)$. A simple band pass filter does not produce a stationary series since there is no $(1-L)^{d}$ term in its numerator. This can produce serious distortions as pointed out by Murray (2000) and Benati (2000) in their studies of ideal band pass filters. Of course a series may be differenced before applying a band pass filter, but again the result may not be what is wanted. If the cycle in the actual series is additive, as in (15), the effect of applying a band pass filter to $d$-th differences is that the gain is multiplied by a factor of $2^{d / 2}(1-\cos \lambda)^{d / 2}$, so low frequencies are attenuated.

To summarise, the proposed generalised low and band pass Butterworth filters are mutually consistent and become closer to ideal filters with rectangular gain functions as $m$ and $n$ tend to infinity. This raises the question of how 
desirable ideal filters are in the first place. In time series modelling the trend index $m$ is usually taken to be one or two. A higher value will give a nonlinear forecast function and an estimated trend which is relatively responsive to shortterm movements in the series. Such trends may not be attractive. On the other hand, higher values of $n$ in the cycle will lead to more concentration on a particular band of frequencies and hence to an extracted cycle which is smoother than would be obtained with the usual value of $n=1$. This may have some merit. To see whether this is the case, the next section investigates how well models with different values of $n$ fit real economic time series.

\section{$5 \quad$ US Macroeconomic time series}

There are two attractions to estimating trends and cycles from a fully specified model. The first is that the implied filters are optimal and mutually consistent for given parameter values. The second is that the parameters can be estimated, so the filters are consistent with the properties of the series. This section demonstrates these points by fitting models to the logarithms of quarterly, seasonally adjusted, US GDP and investment from 1947:1 to 1999:4 based on 1996 price levels (Source: US Dept. of Commerce, Bureau of Economic Analysis: National Accounts Data). The order of the stochastic trend is $m=2$ and interest centres on comparing the results for different orders of the cyclical component.

The estimation results are reported below in table 2 . The calculations were done with a program written in the Ox language of Doornik (1999), with extensive use being made of the state space algorithms in Ssfpack; see appendix and Koopman, Shephard, and Doornik (1999). The parameters were estimated by maximum likelihood and the variances are reported multiplied by $10^{7}$. The equation standard error, $\hat{\sigma}$, has been multiplied by $10^{4}$. The Box-Ljung statistic $Q(P)$ is based on $P$ residual autocorrelations and should be tested against a chi-squared distribution with degrees of freedom equal to $P+1$ minus the number of estimated hyperparameters. Goodness of fit is assessed by $R_{D}^{2}$, the coefficient of determination with respect to first differences, as well as by the equation standard error $\hat{\sigma}$. The figures in table 2 can be compared with those in table 1 which were obtained using the STAMP package of Koopman et al (2000); the small differences can be partly explained by the different ways in which the disturbance terms are set up in the cyclical components. Note that when $\sigma_{\eta}^{2}$ is a free parameter in GDP it is estimated as zero.

\begin{tabular}{lllllllllll} 
Series & Restrictions & $\sigma_{\eta}^{2}$ & $\sigma_{\zeta}^{2}$ & $\sigma_{\kappa}^{2}$ & $\sigma_{\varepsilon}^{2}$ & $\rho$ & $2 \pi / \lambda_{c}$ & $Q(14)$ & $R_{D}^{2}$ & $\sigma$ \\
\hline \hline Real GDP & none & 0 & 17 & 585 & 0 & 0.90 & 17.8 & 17.6 & 0.07 & 99 \\
\hline Investment & none & 21,250 & 0 & 1242 & 337 & 0.98 & 9.6 & 22.6 & 0.14 & 519 \\
& $\sigma_{\eta}^{2}=0$ & 0 & 31 & 20,406 & 0 & 0.87 & 17.6 & 19.1 & 0.08 & 535 \\
\hline
\end{tabular}

Table 1: STAMP parameter estimates and diagostics for US macroeconomic data. 


\begin{tabular}{lllllllllll} 
Series & $n$ & Restrictions & $\sigma_{\zeta}^{2}$ & $\sigma_{\kappa}^{2}$ & $\sigma_{\varepsilon}^{2}$ & $\rho$ & $2 \pi / \lambda_{c}$ & $Q(14)$ & $R_{D}^{2}$ & $\sigma$ \\
\hline \hline Real GDP & 1 & none & 21 & 670 & 0 & 0.92 & 19.2 & 28.3 & 0.05 & 100 \\
& 2 & none & 0 & 594 & 91 & 0.74 & 142.8 & 12.0 & 0.14 & 96 \\
& 2 & $\lambda_{c}$ fixed & 14 & 413 & 117 & 0.76 & 19.2 & 11.2 & 0.09 & 98 \\
\hline Investment & 1 & none & 46 & 23974 & 0 & 0.90 & 20.5 & 36.7 & 0.05 & 544 \\
& 2 & none & 14 & 12126 & 5184 & 0.74 & 21.6 & 17.3 & 0.11 & 527 \\
& 3 & none & 11 & 6074 & 6714 & 0.65 & 19.4 & 12.7 & 0.13 & 523 \\
& 4 & none & 0 & 4564 & 7051 & 0.55 & 22.4 & 13.8 & 0.16 & 514 \\
& 5 & none & 0 & 3159 & 7269 & 0.49 & 20.6 & 13.0 & 0.16 & 513 \\
& 6 & none & 0 & 2379 & 7375 & 0.45 & 19.3 & 12.6 & 0.16 & 513 \\
\hline
\end{tabular}

Table 2: Parameter estimates and diagnostics from fitting models to US macroeconomic series; each variance is multiplied by $10^{7}$ while $2 \pi / \lambda_{c}$ is the period in quarters.

Setting $n=1$ gives estimated periods for both series of around five years; similar results were reported by Harvey and Jaeger (1993) for a shorter sample. For $n=2, \lambda_{c}$ was estimated to be near zero for GDP. This is not very satisfactory, though the Box-Ljung statistic is much lower. However, setting the frequency to the value obtained with $n=1$ gave similar diagnostics to those obtained with unrestricted estimation and only a marginal increase in the equation standard error. The damping factor of the cycle fell from 0.92 to 0.76 . The pattern is similar to the cycle obtained with $n=1$, but it is smoother because some of the high frequency movements have been consigned to the irregular.

Estimation of the investment series for higher values of $n$ was more successful. The period is roughly the same for $n$ from one to six while $\rho$ shows a gradual decline and the irregular variance increases. The cycles are shown for $n=1,2$ and 4 in figures 7 through 9 . The result for $n=6$ is similar to that of $n=4$. Setting $n$ greater than one gives a cycle which is smoother and more clearly defined, thereby enabling turning points to be dated more easily. The higher values of $n$ also point to recent years as belonging to the upswing of a cycle, something which is not apparent when $n=1$.

The implicit weights used by the smoother to extract the trend and cyclethe $w_{j}^{\prime} s$ in expressions like (2)- were calculated with the 'Weights' routine of Koopman and Harvey (1999). These enable a comparison to be made with nonparametric methods in which kernels, or weighting patterns, depend on a pre-assigned bandwidth as opposed to an estimated signal to noise ratio.

The weights for extracting the cycle and trend in US investment near the middle of the sample are shown in figure 10 for $n=1$. The shape of the implicit kernel is determined by the parameter estimates reported earlier for the UC model with a 1st order stochastic cycle. Since there is no irregular, the two contemporaneous weights $(j=0)$ sum to one, and at other points they are equal and opposite. For the cycle, $w_{0}$ exceeds 0.9 indicating an emphasis on the current observation, while the other coefficients show damped oscillations as they collapse toward zero.

When $n=2$, the irregular plays a role and figure 11 shows how smoother cyclical estimates are produced. The current observation receives less weight 


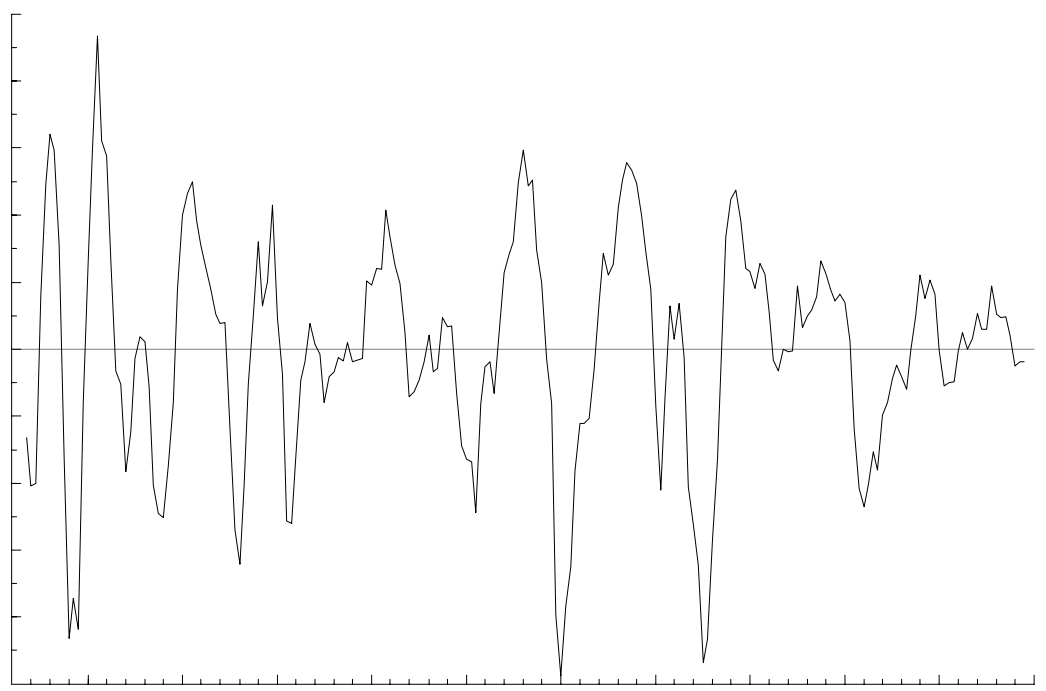

Figure 7: Estimated cycle for US investment (logarithms) with $n=1$.

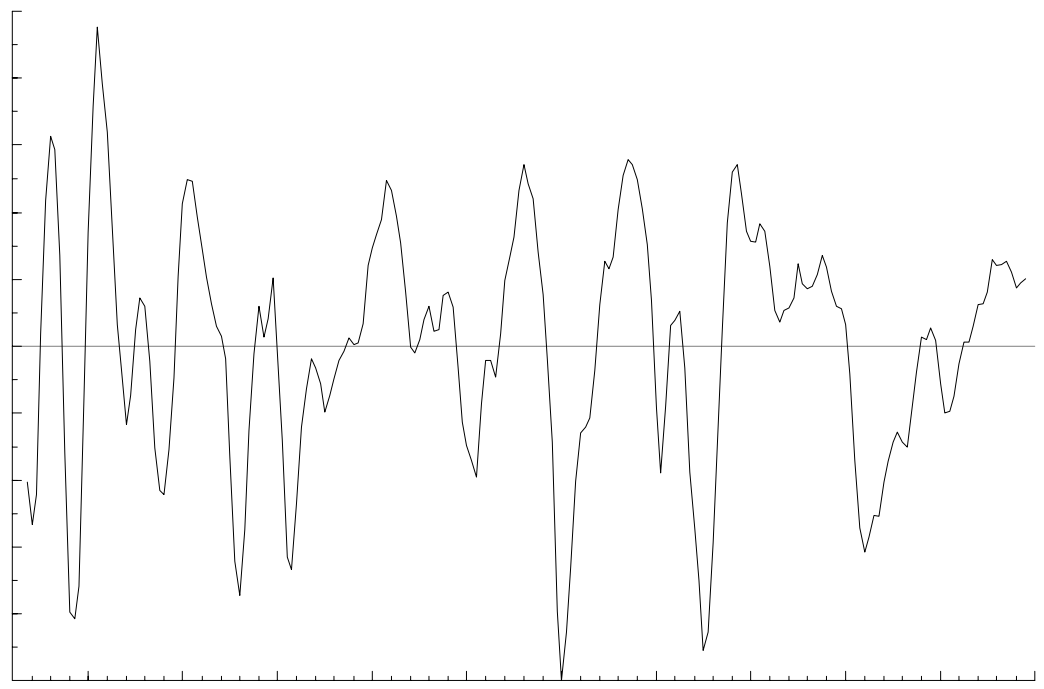

Figure 8: Estimated cycle for US investment (logarithms) with $n=2$. 


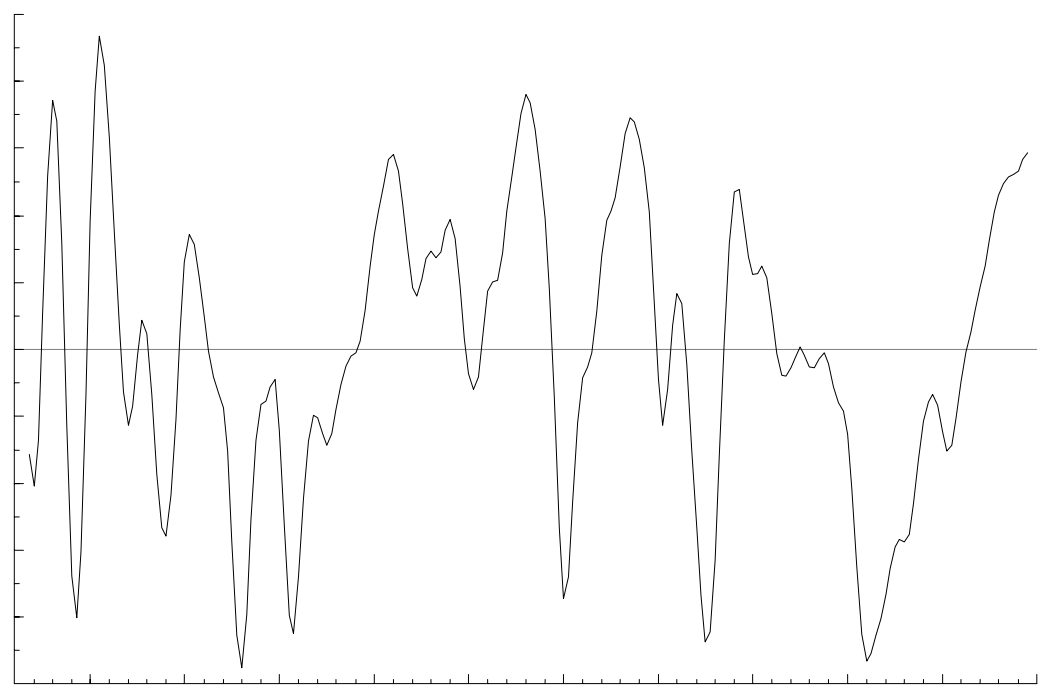

Figure 9: Estimated cycle for US investment (logarithms) with $n=4$.

and the two immediately adjacent observations have a significant positive weighting. Thus the cycle is estimated by averaging over the current observation and the ones immediately adjacent, and then subtracting a weighted sum of the remaining observations.

The weighting pattern for the trend is flatter for $n=2$ as compared with $n=1$, with a somewhat lower peak and no negative weights, at least in the range considered.

The stylised facts from the estimated trends and cycles are such that the trend shows a steady increase in the 1960s and the 1990s. At other times, the underlying growth rate is slower and the cycles are more pronounced. The extracted cycles for US investment in figures 7 through 9 are particularly interesting. As $n$ increases, there are really only two features that change as a smoother cyclical component emerges. First, the peak in the late 1950s becomes lower and second, recent upward movments in the trend become assigned to the cycle. Results for some other countries are presented in Trimbur (2000).

\section{Conclusions}

We have set up a class of unobserved component time series models consisting of stochastic trend, cycle and irregular components. A seasonal component 

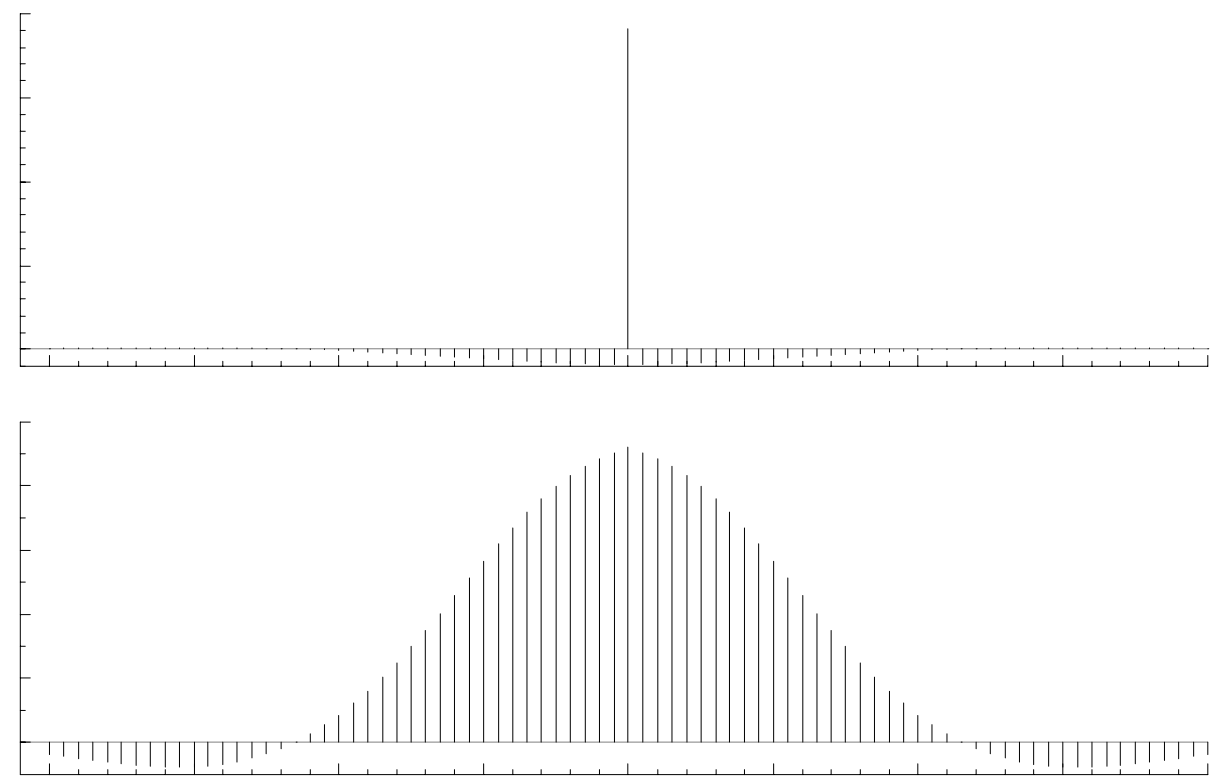

Figure 10: Observation weights for extracting the cycle (top) and the trend (bottom) in US investment for $n=1$. 

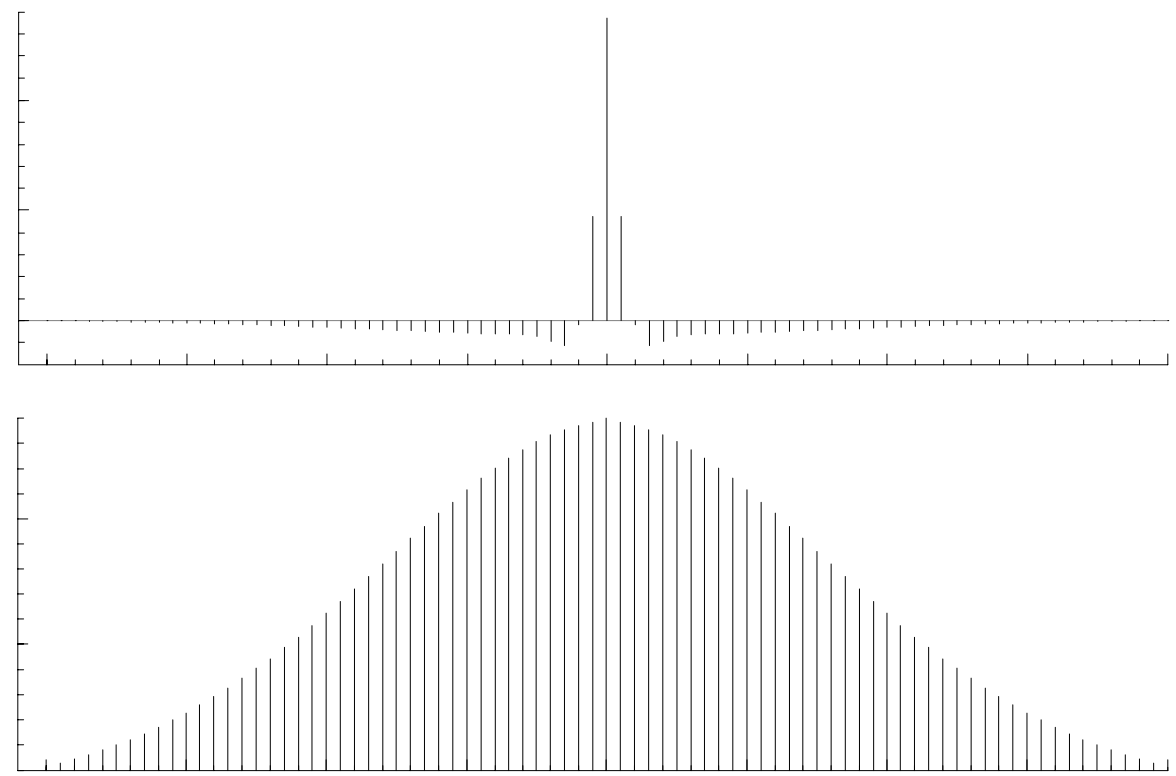

Figure 11: Observation weights for extracting the second-order cycle (top) and the trend (bottom) in US investment. 
may be included if appropriate. The model may be estimated by maximum likelihood and the specification checked by the usual diagnostics. The trend and cycle components are then extracted optimally by the state space smoothing algorithm. The new feature of the model lies in the specification of the cycle which now depends on an index parameter $n$. In previous work $n$ has effectively been set to one, but by allowing a higher value a smoother, more clearly defined, cycle is obtained. The example of US investment illustrates this point with values of $n$ equal to two and four.

The smoothing algorithm applied to a given model implies a particular weighting pattern or filter for the observations. The Wiener-Kolmogorov filter for a doubly infinite sample is easily written down. This enables the model-based smoother to be compared with other filters for extracting trends and cycles. In particular, it transpires that the Butterworth low pass filter is equivalent to trend extraction in a stochastic trend plus noise model, while the Butterworth band pass filter is obtained from a nonstationary cycle plus noise model. By introducing a damping factor which allows the cycle to be stationary, a more general class of Butterworth band pass filters is defined. Setting up a model with both a trend and a cycle leads to a class of generalised Butterworth filters which extract these components in a mutually consistent manner.

The ideal band pass filter can be effectively obtained from the Butterworth band pass filter by setting $n$ to a high value. As has been pointed out, a simple ideal filter can create spurious effects when used directly on a nonstationary economic time series and a generalised band pass Butterworth filter can overcome this problem to some extent by making allowance for the trend. However, the argument for an ideal band pass filter carries across from engineering where one might, for example, be interested in tuning a radio to a particular station. Its use in economics is perhaps problematic, particularly as the implied cyclical model may not be an appealing one.

To summarise, the filters implied by the proposed class of unobserved components models include, as special cases, many filters which are popular in business cycle research. Having a model underpinning a filter solves the problem of how to adapt the weights to the sample end points and how to find mean square errors. Furthermore the model provides insight into when certain filters are appropriate and when they can lead to serious distortions. Our preferred strategy is to avoid potential distortions by using model-based filters which are consistent with the data.

\section{ACKNOWLEDGEMENTS}

Earlier versions of this paper were presented at the Manchester Conference on Growth and Business Cycles in Theory and Practice in July 2000 and at the Summer School in Business Cycles at the European University Institute in Florence in September, 2000. Trimbur wishes to thank the National Science Foundation for financial support. 


\section{References}

Baxter, M., And R. King (1999): "Measuring Business Cycles: Approximate Band-Pass Filters for Economic Time Series," Review of Economics and Statistics, 81, 575-593.

Bell, W. R. (1984): "Signal Extraction for Nonstationary Time Series," Annals of Statistics, 13, 646-664.

Benati, L. (2000): "Band-Pass Filtering, Cointegration, and Business Cycle Analysis," mimeo.

Burns, A. M., And W. C. Mitchell (1946): Measuring Business Cycles. National Bureau of Economic Research.

Christiano, L. J., and T. J. Fitzgerald (1999): "The Band Pass Filter," National Bureau of Economic Research Working Paper, (7257).

Cogley, T., and J. M. Nason (1995): "Cyclical Properties of Baxter-King Filtered Time Series," Journal of Economic Dynamics and Control, 19, 25378.

Doornik, J. A. (1999): Ox: An Object-Oriented Matrix Programming Language. Timberlake Consultants Limited.

Gomez, V. (2000): "The Use of Butterworth Filters for Trend and Cycle Estimation in Economic Time Series," mimeo.

Harvey, A. C. (1989): Forecasting, Structural Time Series Models and the Kalman Filter. Cambridge University Press.

Harvey, A. C., And A. Jaeger (1993): "Detrending and Business Cycle Facts," Journal of Applied Econometrics, 8, 231-247.

Hodrick, R. J., And E. C. PrescotT (1997): "Post-War U.S. Business Cycles: An Empirical Investigation," Journal of Money, Credit, and Banking, $29,1-16$.

Kitagawa, G., And W. Gersch (1996): Smoothness Priors Analysis of Time Series. Springer-Verlag.

Kohn, R., C. Ansley, and C.-H. Wong (1992): "Nonparametric Spline Regression with Autoregressive Moving Average Errors," Biometrika, 79, 33546.

Koopman, S., N. Shephard, and J. Doornik (1999): "Statistical Algorithms for Models in State Space Using SsfPack 2.2," Econometrics Journal, $2,113-166$.

Koopman, S. J., AND A. C. HaRvey (1999): "Computing Observation Weights for Signal Extraction and Filtering," mimeo. 
Murray, C. J. (2000): "Cyclical Properties of Baxter-King Filtered Time Series," mimeo.

Oppenheim, A. V., and R. W. Schaffer (1989): Discrete-Time Signal Processing. Prentice Hall.

Trimbur, T. M. (2000): "Cyclical Components and Signal Extraction in Time Series Models: Generalised Butterworth Filters," mimeo.

Young, P. (1984): Recursive Estimation and Time Series Analysis. SpringerVerlag.

\section{A Appendix: State space form}

The model underlying the generalised Butterworth filter, (15), can be put into state space form (SSF) by defining the $(2 n+m)$ x 1 state vector $\alpha_{t}=\left(\mu_{t}^{\prime}, \psi_{t}^{\prime}\right)^{\prime}$ where

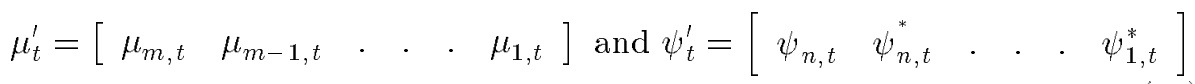

The measurement equation is then

$$
y_{t}=z_{t}^{\prime} \alpha_{t}+\varepsilon_{t}, \quad t=1, \ldots, T,
$$

where the vector $z_{t}$ contains ones in the first and $(m+1)-t h$ positions and zeroes elsewhere. The transition equation for the trend is

$$
\mu_{t}=U_{m} \mu_{t-1}+i_{m} \zeta_{t}
$$

where $U_{m}$ is an $m \times m$ upper triangular matrix of ones and $i_{m}$ is an $m \times 1$ column of ones. The covariance matrix of the disturbance vector is therefore

$$
\operatorname{Var}\left(i_{m} \zeta_{t}\right)=i_{m} i_{m}^{\prime} \sigma_{\zeta}^{2}
$$

The transition equation for the cyclical part of the state vector is

$$
\psi_{t}=T_{\psi} \psi_{t-1}+i_{n} \otimes\left[\begin{array}{c}
\kappa_{t} \\
0
\end{array}\right]
$$

where 


$$
T_{\psi}=U_{n} \otimes\left[\begin{array}{cc}
\rho \cos \lambda_{c} & \rho \sin \lambda_{c} \\
0 & 0
\end{array}\right]+I_{n} \otimes\left[\begin{array}{cc}
0 & 0 \\
-\rho \sin \lambda_{c} & \rho \cos \lambda_{c}
\end{array}\right]
$$

The covariance matrix of the disturbance vector is

$$
\left.\operatorname{Var}\left\{i_{n} \otimes\left[\begin{array}{c}
\kappa_{t} \\
0
\end{array}\right]\right\}\right)=i_{n} i_{n}^{\prime} \otimes\left[\begin{array}{cc}
\sigma_{\kappa}^{2} & 0 \\
0 & 0
\end{array}\right]
$$

The Kalman filter is applied by initialising the nonstationary trend vector, $\mu_{t}$, with a diffuse prior. The unconditional distribution of the cyclical state vector $\psi_{t}$ has mean zero and its covariance matrix $\Gamma$ is found by noting that equation (21) represents a stationary $\operatorname{VAR}(1)$ in $\psi_{t}$. Using a standard formula

$$
v e c\{\Gamma\}=\left[I_{4 n^{2}}-T_{\psi} \otimes T_{\psi}\right]^{-1} v e c\left\{i_{n} i_{n}^{\prime} \otimes\left[\begin{array}{cc}
\sigma_{\kappa}^{2} & 0 \\
0 & 0
\end{array}\right]\right\}
$$

